\title{
Detection of Single Sub-Micrometer Objects of Biological or Technical Origin Using Wide Field Surface Plasmon Microscopy ${ }^{\dagger}$
}

\author{
Shavkat Nizamov, Vitali Scherbahn, Vladimir M. Mirsky * \\ Brandenburgische Technische Universität Cottbus, 01968 Senftenberg, Germany \\ * Correspondence: mirsky@b-tu.de \\ + Presented at the 5th International Symposium on Sensor Science (I3S 2017), Barcelona, Spain, \\ 27-29 September 2017.
}

Published: 6 December 2017

Detection of nano- and microparticles is an important task for chemical analytics, medical diagnostics, food industry, biotechnology, environmental monitoring and many other fields of science and industry. For this purpose, a method based on the detection and analysis of minute signals of surface plasmon resonance images due to adsorption of single particles was developed.

The new technology allows one a real-time detection of interaction of single nano- and microparticles of different origin with sensor surface. Adsorption of each nanoparticle leads to a characteristic diffraction image whose intensity depends on the size and chemical composition of the particle. The number of the nanoparticle - surface binding events per time and surface area characterizes volume concentration. A large monitored surface area of the sensor surface allows one to detect many hundreds events in each frame or totally up to a million particles on the sensor surface, this leads to a very high dynamic range of counting and to a correspondingly high dynamic range in the concentration scale. Depending on the type of particles and experimental conditions, the detection limit for aqueous samples can be below 1000 nanoparticles per microliter. Stable analysis of nanoparticles in very complex environment (fruit juices, wines, cosmetic formulations) was demonstrated.

For determination of chemical composition of single nanoparticles separately, the wide field surface plasmon microscopy can be used as a tandem technique. For example, for analysis of technical nanoparticles it can be assisted by electrochemical analysis. In this case, the gold sensor surface is used both for plasmon microscopy and as a working electrode of electrochemical cell. Applying a linear potential sweep to this electrode, adsorbed nanoparticles can be subjected to an electrochemical conversion leading to the change of their refractive index; the value of electrical potential of this conversion characterizes material of the particular nanoparticle. Notably, such analysis is performed simultaneously but independently for each adsorbed particle.

The method of wide filed surface plasmon microscopy can be applied for ultrasensitive detection and analysis of nano- and microparticles of biological (bacteria, viruses, exosomes), biotechnological, (liposomes, protein nanoparticles for drug delivery) or technical (metallic, oxides, plastic, etc.) origin.

(C) 2017 by the authors. Licensee MDPI, Basel, Switzerland. This article is an open access article distributed under the terms and conditions of the Creative Commons Attribution (CC BY) license (http://creativecommons.org/licenses/by/4.0/). 